\title{
Description of atomic excitations in heavy-ion reactions
}

\author{
J. Reinhardt, B. Müller, and W. Greiner \\ Institut für Theoretische Physik der Johann Wolfgang Goethe-Universität, \\ Robert-Mayer-Strasse 8-10, Postfach 111932 \\ D-6000 Frankfurt-am-Main, West Germany \\ U. Müller \\ Gesellschaft für Schwerionenforschung mbH, Planckstrasse 1, Postfach 110541 , \\ D-6100 Darmstadt 11, West Germany \\ (Received 23 June 1983)
}

\begin{abstract}
Excitations of the atomic shell in heavy-ion collisions are influenced by the presence of a nuclear reaction. In the present Rapid Communication we point out the equivalence between a semiclassical description based on the nuclear autocorrelation function with an earlier model which employs a distribution of reaction times $f(T)$. For the example of $\mathrm{U}+\mathrm{U}$ collisions, results of coupled-channel calculations for positron creation and $K$-hole excitations are discussed for two schematic reaction models.
\end{abstract}

Recently, several suggestions have attracted attention which aim at the determination of nuclear reaction parameters by measuring atomic excitations (cf. Refs. 1-11 and references therein). Among other effects, variations of inner-shell vacancy formation probabilities ${ }^{2,3}$ and oscillations in the spectra of emitted $\delta$ electrons ${ }^{4}$ are expected to arise from interference of excitation taking place before and after the nuclear reaction. Furthermore, the emission of quasimolecular (united atom) $x$ rays $^{5,6}$ and spontaneous positron production ${ }^{7,8}$ in supercritical collisions will be enhanced during the reaction.

These effects are most easily described in a semiclassical model. Here the internuclear motion consists of an incoming and outgoing branch of a classical (usually hyperbolic) trajectory, while the nuclear reaction acts to keep the nuclei close together for an interval of time $T$. If the adiabatic basis is employed the amplitude of an electronic transition $i \rightarrow f$ is (cf. Refs. 3 and 8 )

$$
a_{i f ; T}=\sum_{n n^{\prime}} a_{i n}(0) C_{n n^{\prime}}(T) \tilde{a}_{n^{\prime} f}(\infty),
$$

where (we set $\hbar=1$ )

$$
C_{n n^{\prime}}(T)=\exp \left[-i E_{n}\left(R_{0}\right) T\right] \delta_{n n^{\prime}} .
$$

$a_{\text {in }}(0)$ and $\tilde{a}_{n^{\prime} f}(\infty)$ are the amplitudes for one branch of the trajectory $(-\infty \rightarrow 0$ or $0 \rightarrow+\infty)$ calculated with the energy and angular momentum of the nuclear motion in the ingoing (outgoing) channel. To obtain, e.g., the number of holes in a state $q$, the squared amplitudes (1) have to be summed over all initial states above the Fermi level $F$ and averaged with a distribution function of reaction times $f(T)$ yet to be specified:

$$
P_{q}=\int_{0}^{\infty} d T f(T) \sum_{i>F}\left|a_{i q ; T}\right|^{2},
$$

with the normalization condition

$$
\int_{0}^{\infty} d T f(T)=1 \text {. }
$$

More generally, at a given scattering angle $\theta$ a superposition of both direct (undelayed) scattering and reaction events may be present. For these contributions an incoherent summation was suggested, ${ }^{8}$ leading to an obvious generalization of (3).

Recently, Tomoda and Weidenmüller ${ }^{12,13}$ applied the semiclassical approximation to the full electronic plus nuclear quantum problem assuming that the excitations of both systems take place in separate regions of space. They obtain a formula very similar to (1). Suppressing angular momentum indices the electronic transition amplitude is

$$
a_{i f, N}=\sum_{m} a_{0, i m}(0) S_{0 N}\left(E-E_{m}\right) \tilde{a}_{N, m f}(\infty),
$$

with the nuclear $S$ matrix $S_{0 N}$ for a transition between the states $0 \rightarrow N$. If the $S$ matrix is a randomly fluctuating function of energy, the atomic excitation probability, after averaging over the beam energy, is found to be ${ }^{13}$

$$
\begin{aligned}
P_{q}= & \alpha(\theta) \sum_{i>F}\left|a_{i q}(-\infty,+\infty)\right|^{2} \\
& +[1-\alpha(\theta)] \sum_{i>F} a_{i n} \tilde{a}_{n q} a_{i m}^{*} \tilde{a}_{m q}^{*} A_{m n} .
\end{aligned}
$$

$\alpha(\theta)$ is a measure for the ratio of "direct" and "delayed" reactions and the matrix $A$ is the nuclear autocorrelation function of the fluctuating part of the $S$ matrix:

$$
\left\langle S_{0 N}^{\mathrm{fl}}\left(E^{\prime}\right) S_{0 N}^{\mathrm{ff} *}(E)\right\rangle=\left\langle\left|S_{0 N}^{\mathrm{fl}}(E)\right|^{2}\right\rangle A_{E^{\prime} E} .
$$

In standard compound-nucleus theory,

$$
A_{E^{\prime} E}=\Gamma_{c} /\left[\Gamma_{c}+i\left(E-E^{\prime}\right)\right],
$$

with the compound-nuclear width $\Gamma_{c}$. Here we want to stress that (6) is completely equivalent to the results (1)-(3). Indeed, Eq. (3) can be written in the form

$$
P_{q}=\sum_{i>F} \sum_{n m} a_{i n} \tilde{a}_{n q} a_{i m}^{*} \tilde{a}_{m q}^{*} \int_{0}^{\infty} d T f(T) \exp \left[-i\left(E_{n}-E_{m}\right) T\right] \text {. }
$$

The time distribution function $f(T)$ and the nuclear correlation function $A_{E^{\prime} E}$ are simply related by a Fourier transformation

$$
A_{E+\omega, E}=\int_{0}^{\infty} d T f(T) e^{i \omega T} .
$$


Here we have implemented causality by the condition $f(T)=0$ for $T<0$. This is satisfied if the autocorrelation function is an analytic function in the upper energy halfplane. As is well known, ${ }^{14}$ the assumption of stochastic fluctuations in the $S$ matrix leads to an exponential decay in time. Indeed, the ansatz $f(T)=\Gamma_{c} \exp \left(-\Gamma_{c} T\right)$ leads to (8).

It is envisaged to apply the theory to heavy-ion collisions close to the Coulomb barrier or to deep-inelastic reactions. In both cases it is not clear whether the stochastic arguments leading to the form (8) are well founded. On the contrary, one expects time distributions more or less sharply peaked at a finite time $T_{c}{ }^{15}$ According to (10) this could be transformed to an autocorrelation function exhibiting longrange order. Such an order might be established by the dominant influence of a few collective modes in the nuclear $S$ matrix. This question has direct bearing on experiments looking for structure in the $\delta$-electron spectrum since interference patterns are washed out in the case of (8) (ex- ponential decay). ${ }^{4}$ From precise experimental data, therefore, information may be gained both on the mean time delay (or width $\Gamma_{c}$ ) and on the distribution $f(T)$, and thus on the nuclear autocorrelation function $A_{E^{\prime} E^{\prime}}$.

The equivalence also holds for supercritical collisions ${ }^{16}$ where the matrix $C_{n n}$ of Eq. (2) acquires nondiagonal elements ${ }^{8}$ due to the decay of the $1 s \sigma$ resonance into the adjacent positron continuum $E$. The modified expression for the excitation probability is

$$
P_{q}=\sum_{i>F} \sum_{n n^{\prime}} \sum_{m m^{\prime}} a_{i n} \tilde{a}_{n^{\prime} q} a_{i m}^{*} \tilde{a}_{m^{\prime} q}^{*} M_{n n^{\prime} m m^{\prime}} .
$$

In the case of an exponential time distribution the matrix $M$ is given by

$$
M_{n n^{\prime} m m^{\prime}}=i \Gamma_{c} /\left(E_{m}-E_{n}+i \Gamma_{c}\right) \delta_{n n^{\prime} m m^{\prime}},
$$

where the atomic $1 s$ resonance enters with a complex energy $E_{1 s}-i \Gamma_{0} / 2$. Additional nondiagonal terms arise from the decay to the positron continuum $1 s \rightarrow E_{p}$ :

$$
\begin{aligned}
M_{1 s E_{p} m m^{\prime}}= & \frac{\sqrt{\Gamma_{0} / 2 \pi}}{\left(E_{p}-E_{1 s}+i \Gamma_{0} / 2\right.}\left(\frac{i \Gamma_{c}}{E_{m}-E_{p}+i \Gamma_{c}}-\frac{i \Gamma_{c}}{E_{m}-E_{1 s}-i \Gamma^{\prime}}\right) \delta_{m m^{\prime}}, \\
M_{1 s E_{p} 1 s E_{p}^{\prime}}= & \frac{\sqrt{\Gamma_{0} / 2 \pi}}{E_{p}-E_{1 s}+i \Gamma_{0} / 2} \frac{\sqrt{\Gamma_{0} / 2 \pi}}{E_{p}^{\prime}-E_{1 s}-i \Gamma_{0} / 2} \\
& \times\left(\frac{i \Gamma_{c}}{E_{p}^{\prime}-E_{p}+i \Gamma_{c}}-\frac{i \Gamma_{c}}{E_{p}^{\prime}-E_{1 s}+i \Gamma^{\prime}}-\frac{i \Gamma_{c}}{E_{1 s}-E_{p}+i \Gamma^{\prime}}+\frac{\Gamma_{c}}{\Gamma_{0}+\Gamma_{c}}\right),
\end{aligned}
$$
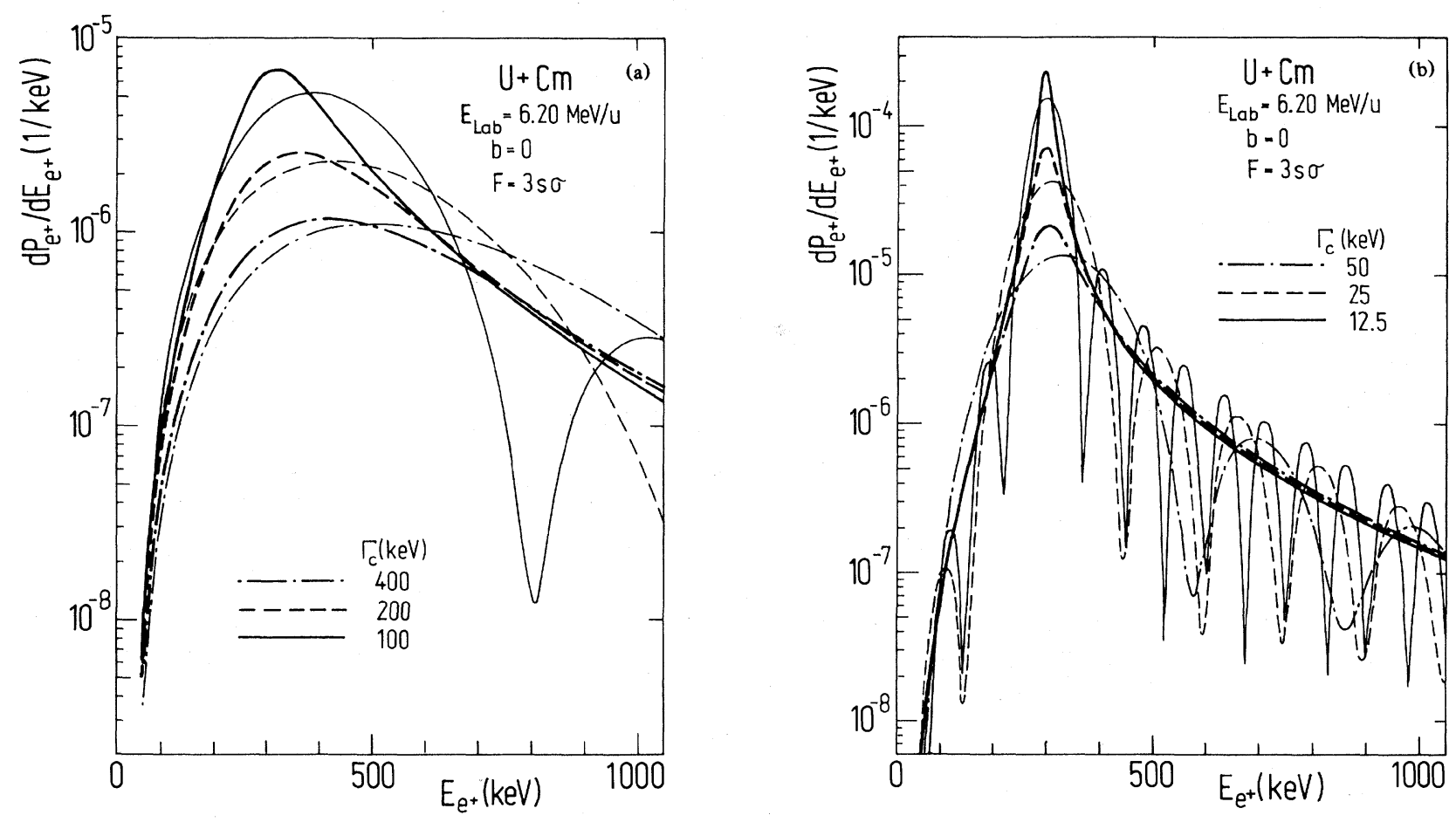

FIG. 1. Spectra of positrons emitted in the $s_{1 / 2}$ channel in supercritical $\mathrm{U}+\mathrm{Cm}$ collisions at $6.2-\mathrm{MeV} / \mathrm{u}$ impact energy, calculated under the assumption that a nuclear reaction takes place. Predictions for an exponential (full lines) and a sharp (thin lines) distribution of reaction times, i.e., $f(T)=\Gamma_{c} \exp \left(-\Gamma_{c} T\right)$ and $f(T)=\delta\left(T-1 / \Gamma_{c}\right)$, are compared. (a) Nuclear widths $\Gamma_{c}=400,200$, and $100 \mathrm{keV}$. (b) $\Gamma_{c}=50$, 25 , and $12.5 \mathrm{keV}$. 

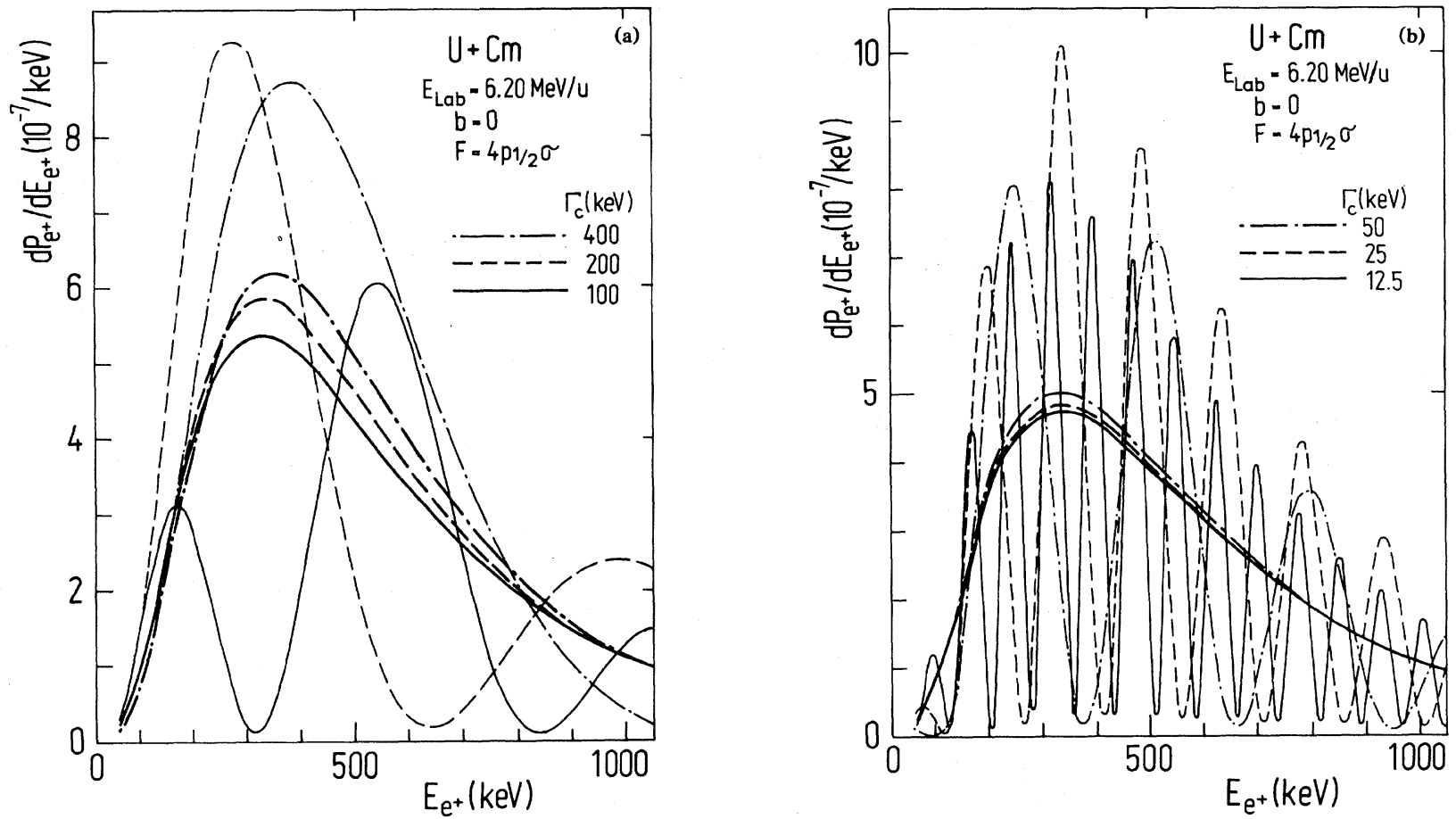

FIG. 2. Same as Fig. 1 for the subcritical $p_{1 / 2}$ states. For the exponential time distribution the spectrum is smooth and changes only weakly with $\Gamma_{c}$.
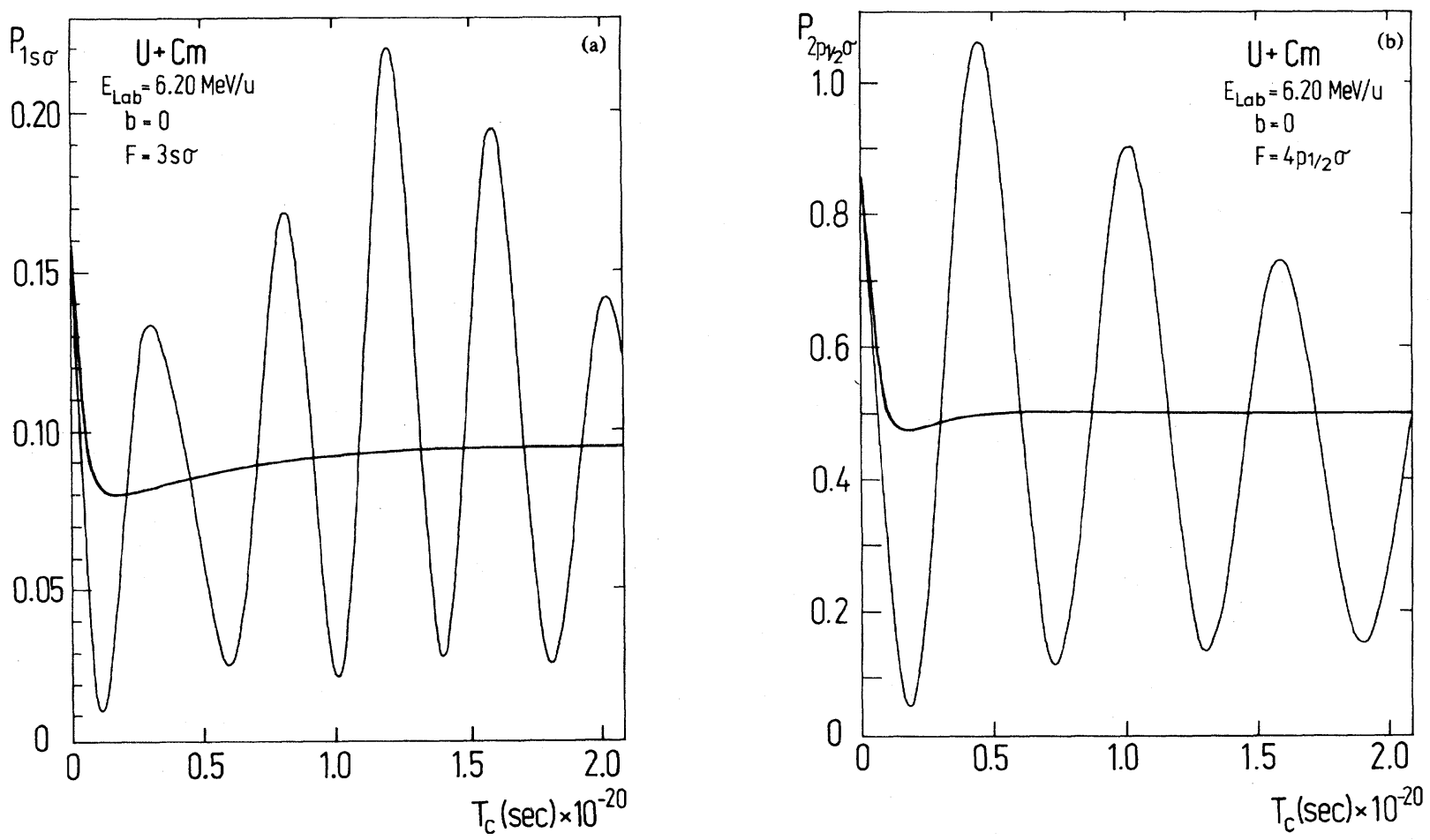

FIG. 3. Probability for exciting $K$ vacancies in a $\mathrm{U}+\mathrm{Cm}$ reaction as a function of the nuclear delay time (inverse width) $T_{c}$. 
where $\Gamma^{\prime}=\Gamma_{c}+\Gamma_{0} / 2$ and

$$
M_{n n^{\prime} m m^{\prime}}=M_{n^{\prime} n m m^{\prime}}=M_{m m^{\prime} n n^{\prime}}^{*} \text {. }
$$

In (13) the decay matrix element was approximated by a constant $\Gamma\left(E_{p}\right)=\Gamma_{0}$, and coupling between different positron states was neglected.

Let us examine the consequences for the positron spectrum in supercritical collisions. Leaving aside positron creation outside the reaction region entirely (which is not a good approximation, however, except for very long reaction times), the combined Eqs. (11) to (13) yield, to a BreitWigner-shaped spectrum,

$$
\frac{d P}{d E} \simeq P_{1 s}(0)(2 \pi)^{-1} \frac{\Gamma_{0}}{\left(\Gamma_{c}+\Gamma_{0}\right)} \frac{2 \Gamma^{\prime}}{\left(E-E_{1 s}\right)^{2}+\Gamma^{\prime 2}} .
$$

As a result of the presence of large values of $T$ in the time integral (3) the resulting "positron line" is clearly narrower compared with the case of a sharp time distribution $f(T)=\delta\left(T-T_{c}\right)$ considered previously ${ }^{8}$ if one identifies $T_{c}$ with the inverse nuclear width $1 / \Gamma_{c}$. While the linewidth full width at half maximum, according to the simple analytic expression (14), is $\Delta E=2 \Gamma^{\prime}=2 \Gamma_{c}$, the sharp time distribution leads to $\Delta E \simeq 5.56 \Gamma_{c}$. The effect of averaging over a distribution of delay times is demonstrated quantitatively by the coupled-channel calculations of Fig. 1. Here the spectra of positrons emitted in the angular momentum channel $s_{1 / 2}$ have been calculated for $\mathrm{U}+\mathrm{Cm}$ collisions at $6.2-\mathrm{MeV} / \mathrm{u}$ bombarding energy. For several values of the nuclear width $\Gamma_{c}$ (inverse reaction time) between 400 and $12.5 \mathrm{keV}$ the predictions of the sharp (bold lines) and exponential (thin lines) distribution functions are compared. While the shapes of the corresponding spectra for the two models differ quite distinctly it is not immediately clear how much information on the distribution function $f(T)$ can be gained from the experiment. Additional broadening and distortion of the line will result from experimental effects (Doppler broadening). Also, the position of the line is very sensitive to the nuclear configuration during the reaction. Therefore a realistic reaction model probably will produce a superposition of lines centered at various (neighboring) energies making the analysis more complex.

Figure 2 , in addition, shows the emission of $p_{1 / 2}$ positrons. Since these states are subcritical the time delay does not result in an enhancement of the probability but only in interferences which are completely smeared out by an exponential distribution $f(T)$.

A similar effect is found for $K$-hole production. Figure 3 shows the probability of $1 s \sigma$ and $2 p_{1 / 2} \sigma$ vacancies as a function of $T_{c}$ (or $1 / \Gamma_{c}$ ). By use of the exponential distribution the oscillatory structure of $P\left(T_{c}\right)$ is lost. The sharp decrease due to destructive interference at rather small values of $T_{c}$, however, survives the folding and should be observable experimentally.

In summary, we have shown the equivalence between two models for electronic excitations associated with a nuclear reaction, one formulated in terms of the nuclear autocorrelation function, the other using a distribution of delay times. While the former representation is more directly related to microscopic quantities, i.e., the nuclear $S$ matrix, the latter has the advantage of a clearer intuitive interpretation.
${ }^{1}$ E. Merzbacher, in X-ray and Atomic Inner-Shell Physics-1982, edited by B. Crasemann, AIP Conf. Proc. No. 94 (AIP, New York, 1982), p. 1; W. Meyerhof, ibid., p. 13.

${ }^{2}$ R. Anholt, Phys. Lett. 88B, 262 (1979).

${ }^{3}$ U. Müller, J. Reinhardt, G. Soff, and W. Greiner, Z. Phys. A 297, 357 (1980).

${ }^{4}$ G. Soff, J. Reinhardt, B. Müller, and W. Greiner, Phys. Rev. Lett. 43, 1981 (1979).

5R. Anholt, Z. Phys. 292, 123 (1979).

${ }^{6} \mathrm{~J}$. Kirsch, B. Müller, and W. Greiner, Phys. Lett. 94A, 151 (1983).

${ }^{7}$ J. Rafelski, B. Müller, and W. Greiner, Z. Phys. A 285, 49 (1978).

${ }^{8}$ J. Reinhardt, U. Müller, B. Müller, and W. Greiner, Z. Phys. A $\underline{303}, 173$ (1981).
9J. S. Blair and R. Anholt, Phys. Rev. A 25, 907 (1982).

${ }^{10}$ J. M. Feagin and L. Kocbach, J. Phys. B 14, 4349 (1981).

${ }^{11}$ U. Heinz, B. Müller, and W. Greiner, University of Frankfurt Report No. 106/1983 [Ann. Phys. (N.Y.) (in press)].

${ }^{12}$ T. Tomoda, Max Planck Institut Heidelberg Report No. MPI H1983-V8 (unpublished).

${ }^{13}$ T. Tomoda and H. A. Weidenmüller, Max Planck Institut Heidelberg Report No. MPI H-1983-V6 [Phys. Rev. C (in press)].

${ }^{14}$ S. Yoshida, Annu. Rev. Nucl. Sci. 24, 1 (1974).

${ }^{15} \mathrm{~A}$. Gobbi and W. Nörenberg, in Heavy Ion Collisions, Vol. 2, edited by R. Bock (North-Holland, Amsterdam, 1980).

16J. Reinhardt, B. Müller, and W. Greiner, Phys. Rev. A 24, 103 (1981). 